\title{
ZERO-HOPF POLYNOMIAL CENTERS OF THIRD-ORDER DIFFERENTIAL EQUATIONS
}

\author{
ISAAC A. GARCÍA ${ }^{1}$ AND CLAUDIA VALLS ${ }^{2}$
}

\begin{abstract}
We study the 3-dimensional center problem at the zero-Hopf singularity in some families of polynomial vector fields arising from third-order polynomial differential equations. After proving some general properties we check that the quadratic family has no 3-dimensional centers. Later we characterize all the 3dimensional centers in the cubic homogeneous family. Finally we give a partial classification of the 3 -dimensional centers at just one singularity of the full cubic family and propose one open problem to close this classification.
\end{abstract}

\section{IntRoduction AND STATEMENT OF THE MAIN RESUlts}

The Kukles systems are described by second-order ordinary differential equations $\ddot{x}=g_{3}(x, \dot{x})$ where $g_{3} \in \mathbb{R}_{3}[x, \dot{x}]$ is a real cubic polynomial. They were named like this since they were first studied by the Russian mathematician I. S. Kukles in [5]. The over dote denotes, as usual, derivative with respect to the time independent variable $t$. When $g_{3}(x, y)=-x+\cdots$ in [5] it is investigated the center problem at the monodromic singularity $(x, y)=(0,0)$ of its associated planar vector field $\dot{x}=y, \dot{y}=g_{3}(x, y)$. Due to both theoretical and practical applications several papers have been published on the center problem for this kind of cubic systems, see for example $[2,10]$. We recall that the characterization of the centers in analytic families of planar vector fields began with the pioneering works of Poincaré [8] and Liapunov [6]. See also the the book [9] for a modern approach based on computational algebra techniques.

In this paper we consider a more general situation, by considering higher-order Kukles systems. Indeed, we consider third-order ordinary differential equations

$$
\dddot{x}=f_{n}(x, \dot{x}, \ddot{x})
$$

2010 Mathematics Subject Classification. 37G15, 37G10, 34C07.

Key words and phrases. Zero-Hopf singularity, three-dimensional vector fields, continua of periodic orbits, Poincaré map. 
with $f_{n} \in \mathbb{R}_{n}[x, \dot{x}, \ddot{x}]$, a real polynomial in three variables of degree $n$.

We transform the differential equation (1) in the usual way as a polynomial differential system

$$
\dot{x}=y, \dot{y}=z, \dot{z}=f_{n}(x, y, z) .
$$

A real singularity $(x, y, z)=\left(x_{0}, 0,0\right) \in \mathbb{R}^{3}$ of $(2)$ is called a zero-Hopf singularity if its associated eigenvalues are $\{ \pm i, 0\}$ with $i^{2}=-1$. We say that a zero-Hopf singularity is a 3-dimensional center if there is a neighborhood of it in $\mathbb{R}^{3}$ completely foliated by periodic orbits of (2), including continua of equilibria as trivial periodic orbits. As far as we know the only work that completely addresses this issue is [4] although in [7] it also appears in the context of the complete analytic local integrability at the zero-Hopf singularity.

The main results of this paper are the following. First we see how a discrete symmetry acts on any zero-Hopf singularity of (2) producing a 3-dimensional center.

Theorem 1. If $f_{n}(x,-y, z)=-f_{n}(x, y, z)$ then any zero-Hopf singularity of (2) is a 3-dimensional center.

The next result shows one reduction from the 3-dimensional center problem to the classical nondegenerate center problem of a planar vector field.

Theorem 2. Let the origin be a zero-Hopf singularity of (2) with $\frac{\partial f_{n}}{\partial x} \equiv$ 0 . If the planar system $\dot{y}=z, \dot{z}=f_{n}(y, z)$ has a center at the origin in $\mathbb{R}^{2}$ then the origin in $\mathbb{R}^{3}$ is a 3-dimensional center of (2).

Next result tell us that the minimum degree $n$ needed for the appearance of 3-dimensional centers in family (2) is $n=3$.

Theorem 3. The quadratic $(n=2)$ vector field (2) has no 3-dimensional centers.

Now we present the classification of all the 3-dimensional centers of family (2) when $n=3$ and $f_{3}$ is a cubic homogeneous polynomial.

Theorem 4. The cubic vector field (2) with $f_{3}(x, y, z)$ a cubic homogeneous polynomial satisfies the following:

(i) If $f_{3}(x, 0,0) \not \equiv 0$ then there is no zero-Hopf singularity in the whole family (2).

(ii) When $f_{3}(x, 0,0) \equiv 0$ family (2) has a 3-dimensional center if and only if it has the form

$$
\dot{x}=y, \dot{y}=z, \dot{z}=-K^{2} x^{2} y+A y^{3}+B x y z+C y z^{2}
$$

with $K \neq 0$. Actually it has a continuum of 3-dimensional centers at any point of the $x$-axis except the origin. 
When $n=3$ and the origin is a zero-Hopf singularity of (2) with spectrum $\{ \pm i \omega, 0\}$ and $\omega \neq 0$, the cubic polynomial $f_{3}$ is written as $f_{3}(x, y, z)=-\omega^{2} y+\hat{f}_{3}(x, y, z)$ with $\hat{f}_{3}$ a polynomial of degree 3 without constant and linear terms. Performing a linear change of variables and a time rescaling $t \mapsto t / \omega$ the linear part of system (2) is written in real Jordan canonical form and (2) becomes

$$
\dot{x}=-y+\omega^{2} \mathcal{F}(x, y, z), \dot{y}=x, \dot{z}=\mathcal{F}(x, y, z)
$$

where $\omega^{3} \mathcal{F}(x, y, z)=\hat{f}_{3}\left(-x / \omega^{2}+z, y / \omega, x\right)$.

Now we give a partial classification of the 3 -dimensional centers at the origin of the full cubic $(n=3)$ family $(2)$.

Theorem 5. The cubic $(n=3)$ family (2) has a 3-dimensional center at the origin when the function $\mathcal{F}(x, y, z)$ of its associated family (4) is of the form:

(i) $\omega^{2} \mathcal{F}(x, y, z)=F x^{2}+C x y-F y^{2}+G x^{2} z+D x y z-G y^{2} z$ with $D F-C G=0$;

(ii) $\omega^{2} \mathcal{F}(x, y, z)=C x y+H x^{2} y-\frac{H}{3} y^{3}-\frac{4}{3} H \omega^{2} x y z$;

(iii) $\omega^{2} \mathcal{F}(x, y, z)=H x^{2} y-\frac{H}{3} y^{3}+G x^{2} z+D x y z-G y^{2} z$ with $G H=0$;

(iv) $\omega^{2} \mathcal{F}(x, y, z)=C x y+H x^{2} y-H y^{3}-H \omega^{2} x y z$ with $H \neq 0$;

(v) $\omega^{2} \mathcal{F}(x, y, z)=C x y+H x^{2} y-\frac{1}{2} H \omega^{2} x y z$ with $H \neq 0$;

(vi) $\omega^{2} \mathcal{F}(x, y, z)=\left(\frac{2 D}{\omega^{2}}+H\right) y^{3}+H x^{2} y+D x y z$;

(vii) $\omega^{2} \mathcal{F}(x, y, z)=C x y+\frac{A}{\omega^{2}} y z+H x^{2} y+\frac{B}{\omega^{4}} y^{3}+D x y z+\frac{E}{\omega^{2}} y z^{2}$.

Moreover if the origin is a 3-dimensional center then $\mathcal{F}(x, y, z)$ is either of the above forms or has the expressions:

(viii) $\omega^{2} \mathcal{F}(x, y, z)=F x^{2}+C x y-F y^{2}+I x^{3}+H x^{2} y-3 I x y^{2}-\frac{H}{3} y^{3}-$ $\frac{3}{2} I \omega^{2} x^{2} z-H \omega^{2} x y z+\frac{3}{2} I \omega^{2} y^{2} z$ with $2 F H-3 C I=0 ;$

(ix) $\omega^{2} \mathcal{F}(x, y, z)=F x^{2}-\frac{D F}{I K^{2}} x y-F y^{2}+I x^{3}+H x^{2} y-3 I x y^{2}+$ $\left(\frac{2 D}{\omega^{2}}+H\right) y^{3}-I \omega^{2} x^{2} z+D x y z+I \omega^{2} y^{2} z$ with $I \neq 0$ and $2 D^{2}+$ $3 D H K^{2}+H^{2} K^{4}-I^{2} K^{4}=0$;

(x) $\omega^{2} \mathcal{F}(x, y, z)=F x^{2}+C x y-F y^{2}+H x^{2} y-\frac{A F}{\omega^{4}} x y^{2}+\frac{A}{\omega^{2}} y z-$ $H \omega^{2} x y z+\frac{A F}{\omega^{2}} y^{2} z$;

Remark 6. We have proved that family (4) with the $\mathcal{F}(x, y, z)$ given in the statement (viii) of Theorem 5 has a 3 -dimensional center at the origin in the parameter case $I=0$. Otherwise if $I \neq 0$ then we have also showed that after a translation in the $z$-axis we can assume the parameters $F=C=0$ in the expression of such a $\mathcal{F}$ without loss of generality.

On the other hand we have also checked that the origin of (4) with the $\mathcal{F}(x, y, z)$ stated in part (x) of Theorem 5 is a 3 -dimensional center when the parameters are either $F=0$ or $A=H=0$. 
All together Theorem 5 and Remark 6 implies that we will have the complete classification of the 3 -dimensional centers at the origin in the cubic family (2) if and only if we can solve the next problem.

Open Problem Find out if the origin is a 3-dimensional center for family (4) where $\mathcal{F}(x, y, z)$ is given by one of the following tree cases of Theorem 5: (viii) with $I \neq 0$ and $F=C=0$; (ix); (x) with either $F \neq 0$ or $A^{2}+H^{2} \neq 0$.

In analogy with the classical two-dimensional center problem in the qualitative theory of differential equations, the necessary 3-dimensional center conditions in the parameter space for family (4) are derived by vanishing the initial coefficients $\delta_{j}$ of the series of an adequate Poincaré map, see the next section. But the sufficient 3-dimensional center conditions (which guarantee that actually $\delta_{j} \equiv 0$ for any $j \in \mathbb{N}$ ) in the forthcoming proof of Theorem 5 needs the use of symmetryintegrability arguments. The technical difficulties why the cases stated in the above open problem cannot be solved are that, although we have checked that $\delta_{j} \equiv 0$ for $1 \leq j \leq 11$ so that it is very probable that the singularity is a 3-dimensional center, we have not been successful in finding the necessary symmetries or first integrals that ensure the existence of that 3-dimensional center.

\section{The BACKGround AND SOME AUXILIARY RESUlts}

2.1. The Poincaré map at the zero-Hopf singularity. In this subsection we present a brief description of the theory introduced in [4] and next developed in [3]. There, it is considered an analytic threedimensional system

$$
\begin{aligned}
& \dot{x}=-y+F_{1}(x, y, z) \\
& \dot{y}=x+F_{2}(x, y, z) \\
& \dot{z}=F_{3}(x, y, z),
\end{aligned}
$$

defined on a neighborhood $\mathcal{U} \subset \mathbb{R}^{3}$ of the origin and having a zero-Hopf singularity at the origin. Then, the $F_{i}$ are real analytic functions on $\mathcal{U}$ without independent and linear terms.

Theorem $7([4,3])$. We consider system (5) defined on a neighborhood $\mathcal{U} \subset \mathbb{R}^{3}$ of the origin. Let $\delta>0$ be sufficiently large but fixed and define $\mathcal{C}_{\delta}=\left\{(x, y, z) \in \mathcal{U}: z^{2}>\delta\left(x^{2}+y^{2}\right)\right\}$, a thin solid cone with vertex at the origin surrounding the $z$-axis. Doing first the rescaling $(x, y, z) \mapsto$ $(x / \varepsilon, y / \varepsilon, z / \varepsilon)$ and later the polar blow-up $(x, y, z) \mapsto(\theta, r, w)$ defined 
by

$$
x=r \cos \theta, y=r \sin \theta, z=r w,
$$

system (5) can be written in $\mathcal{U} \backslash \mathcal{C}_{\delta}$, for $|r|$ and $|\varepsilon|$ sufficiently small, as the analytic system

$$
\frac{d r}{d \theta}=\varepsilon R(\theta, r, w ; \varepsilon), \quad \frac{d w}{d \theta}=\varepsilon W(\theta, r, w ; \varepsilon),
$$

with invariant set $\{r=0\}$ and defined on the cylinder $\{(\theta, r, w) \in$ $\left.\mathbb{S}^{1} \times \mathbb{R} \times \mathcal{K}\right\}$ where $\mathbb{S}^{1}=\mathbb{R} / 2 \pi \mathbb{Z}$ and $\mathcal{K}=\left\{w \in \mathbb{R}:|w| \leq \delta^{2}\right\}$.

Denoting by $\Psi\left(\theta ; r_{0}, w_{0} ; \varepsilon\right)=\left(r\left(\theta ; r_{0}, w_{0} ; \varepsilon\right), w\left(\theta ; r_{0}, w_{0} ; \varepsilon\right)\right)$ the solution of $(7)$ satisfying the initial condition $\Psi\left(0 ; r_{0}, w_{0} ; \varepsilon\right)=\left(r_{0}, w_{0}\right) \in$ $\mathbb{R} \times \mathcal{K}$ for $\left|r_{0}\right|$ sufficiently small, one can define the Poincaré translation map $\Pi\left(r_{0}, w_{0} ; \varepsilon\right)=\Psi\left(2 \pi ; r_{0}, w_{0} ; \varepsilon\right)$ and the analytic displacement map $d\left(r_{0}, w_{0} ; \varepsilon\right)=\Pi\left(r_{0}, w_{0} ; \varepsilon\right)-\left(r_{0}, w_{0}\right)$.

Remark 8. The need to restrict the values of $w$ to the arbitrary but fixed compact set $\mathcal{K}$ containing the origin is clarified in [3]. From the geometry associated to the polar blow-up (6), we see that $(x, y, z) \in$ $\mathcal{U} \backslash \mathcal{C}_{\delta}$ when $w \in \mathcal{K}$ and that (6) is a diffeomorphism in $\mathcal{U} \backslash \mathcal{C}_{\delta}$. Although the polar blow-up (6) does not cover $\mathcal{U}$, we emphasize that any periodic orbit of (5) in $\mathcal{U}$ not intersecting the $z$-axis is contained in $\mathcal{U} \backslash \mathcal{C}_{\delta}$ for $\delta$ sufficiently large. In consequence, the zeros of the displacement map $d\left(r_{0}, w_{0}\right)$, with $w_{0} \in \mathcal{K}$ and $\left|r_{0}\right| \ll 1$, pick up all these periodic orbits. Anyway, see the arguments of [3] based on the properties real analytic functions of several variables that vanish on a set of positive measure, in [3] it is proved that the origin of system (5) is a 3-dimensional center if and only if $d\left(r_{0}, w_{0} ; \varepsilon\right) \equiv 0$.

The idea behind the rescaling $(x, y, z) \mapsto(x / \varepsilon, y / \varepsilon, z / \varepsilon)$ in Theorem 7 is that now we can compute the Taylor series about $\varepsilon=0$

$$
d\left(r_{0}, w_{0} ; \varepsilon\right)=\sum_{j \geq 1} \delta_{j}\left(r_{0}, w_{0}\right) \varepsilon^{j}
$$

such that condition $d\left(r_{0}, w_{0} ; \varepsilon\right) \equiv 0$ is equivalent to the vanish of all the coefficients $\delta_{j}\left(r_{0}, w_{0}\right)$. Using the terminology of the analogous twodimensional problems in the qualitative theory of differential equations (see for example [1]), we will referer to the analytic function $\delta_{j}: \mathbb{R}^{2} \rightarrow$ $\mathbb{R}^{2}$ as the $j$-th Melnikov function.

The computation of the Melnikov functions $\delta_{j}$ is algorithmic although aid of an algebraic manipulator is highly recommended because the calculations involved are massive. First we expand in power series 
of $\varepsilon$ both system (7) and its solution. Then we have

$$
\begin{aligned}
& \frac{d r}{d \theta}=\varepsilon R(\theta, r, w ; \varepsilon)=\varepsilon \sum_{j \geq 1} R_{j}\left(\theta ; r_{0}, w_{0}\right) \varepsilon^{j} \\
& \frac{d w}{d \theta}=\varepsilon W(\theta, r, w ; \varepsilon)=\varepsilon \sum_{j \geq 1} W_{j}\left(\theta ; r_{0}, w_{0}\right) \varepsilon^{j}
\end{aligned}
$$

and

$$
\Psi\left(\theta ; r_{0}, w_{0} ; \varepsilon\right)=\left(r_{0}, w_{0}\right)+\left(\sum_{j \geq 1} \Psi_{1, j}\left(\theta ; r_{0}, w_{0}\right) \varepsilon^{j}, \sum_{j \geq 1} \Psi_{2, j}\left(\theta ; r_{0}, w_{0}\right) \varepsilon^{j}\right) .
$$

Since $\Psi$ satisfies the initial condition $\Psi\left(0 ; r_{0}, w_{0} ; \varepsilon\right)=\left(r_{0}, w_{0}\right)$ it follows that $\Psi_{i, j}\left(0 ; r_{0}, w_{0}\right)=0$ for all $j \in \mathbb{N}$ and $i \in\{1,2\}$. In summary the displacement map is

$$
\begin{aligned}
d\left(r_{0}, w_{0} ; \varepsilon\right) & =\Psi\left(2 \pi ; r_{0}, w_{0} ; \varepsilon\right)-\left(r_{0}, w_{0}\right) \\
& =\sum_{j \geq 1}\left(\Psi_{1, j}\left(2 \pi ; r_{0}, w_{0}\right), \Psi_{2, j}\left(2 \pi ; r_{0}, w_{0}\right)\right) \varepsilon^{j},
\end{aligned}
$$

and consequently the $j$-th Melnikov function is

$$
d_{j}\left(r_{0}, w_{0}\right)=\left(\Psi_{1, j}\left(2 \pi ; r_{0}, w_{0}\right), \Psi_{2, j}\left(2 \pi ; r_{0}, w_{0}\right)\right) .
$$

2.2. Generalized reversibility and local analytic first integrals near equilibriums. In this subsection we summarize some results obtained in [7] that we will need later in the proof of Theorem 1. The framework is to detect analytic first integrals near equilibrium points (located at the origin) of analytic vector fields

$$
\dot{\mathbf{x}}=f(\mathbf{x})=A \mathbf{x}+\cdots
$$

in some open neighborhood of the origin in $\mathbb{C}^{n}$. The main argument is the well-known fact that any formal first integral of a (formal) PoincaréDulac normal form

$$
\dot{\mathbf{x}}=\hat{f}(\mathbf{x})=A \mathbf{x}+\cdots
$$

of (9) is also a first integral of the linear system

$$
\dot{\mathrm{x}}=A_{s} \mathbf{x}
$$

where $A_{s}$ is the semisimple part of the linearization $A$ of (9). Recall that we can always decompose $A=A_{s}+A_{n}$ with $A_{S}$ semisimple (diagonalizable in $\mathbb{C}$ ) and $A_{n}$ is nilpotent with commutation $\left[A_{s}, A_{n}\right]=0$. Also we remind that the formal vector field (10) is in Poincaré-Dulac normal form if $\left[A_{s}, \hat{f}\right]=0$.

In this context a very interesting case to investigate is whether all formal first integrals of $A_{s}$ are conserved by a normal form because this property may ensure convergence of the normal form and also complete 
local analytic integrability. The following result was first proven by Zhang [11, 12], see also Theorem 9 in [7].

Theorem $9([11,12]$ and $[7])$. Assume that $A_{s} \neq 0$ and the linear system (11) in $\mathbb{C}^{n}$ admits $n-1$ independent polynomial first integrals. If some formal Poincaré-Dulac normal form of (9) admits n-1 independent formal first integrals, then (9) admits a convergent transformation to Poincaré-Dulac normal form and also it possesses $n-1$ independent analytic first integrals.

Now we adapt the general Proposition 11 of [7] to our purpose, hence we state it in the very particular case that we need. We say that (9) is time-reversible if there is a nonsingular $n \times n$ matrix $T$ such that $T^{-1} \circ f \circ T=-f$.

Proposition 10 ([7]). Consider system (9) with $n=3$ and diagonal linear part $A=A_{s}$ with spectrum $\{ \pm i \omega, 0\}$ where $\omega \in \mathbb{R} \backslash\{0\}$. If (9) is time-reversible then the two independent first integrals of (11) are conserved in a Poincaré-Dulac normal form of (9).

\section{Proof of Theorem 1}

First we observe that any real singularity $\left(x_{0}, 0,0\right)$ of $(2)$ can be placed at the origin via the translation $(x, y, z) \mapsto\left(x-x_{0}, y, z\right)$ without affecting the form of (2). Hence without loss of generality we will assume that the origin is a zero-Hopf singularity of (2). In particular $f_{n}(x, y, z)=-\omega^{2} y+\hat{f}_{n}(x, y, z)$ with $\omega \neq 0$ and $\hat{f}_{n}$ a polynomial of degree $n$ without constant nor linear terms such that the eigenvalues of the linearization at the origin of $(2)$ are $\{ \pm i \omega, 0\}$. Moreover we can perform a linear change of variables $(x, y, z)^{T} \mapsto P^{-1}(x, y, z)^{T}$ where

$$
P=\left(\begin{array}{ccc}
-\frac{1}{\omega^{2}} & 0 & 1 \\
0 & \frac{1}{\omega} & 0 \\
1 & 0 & 0
\end{array}\right)
$$

and a time rescaling $t \mapsto t / \omega$ such that the linear part of system (2) is written in real Jordan canonical form. In short, after all these transformations (2) becomes

$$
\begin{aligned}
& \dot{x}=-y+\omega^{2} \mathcal{F}(x, y, z) \\
& \dot{y}=x \\
& \dot{z}=\mathcal{F}(x, y, z)
\end{aligned}
$$

where $\mathcal{F}(x, y, z)=\frac{1}{\omega^{3}} \hat{f}_{n}\left(-x / \omega^{2}+z, y / \omega, x\right)$. 
We also can transform system (12) via the linear complex change of variables $(x, y, z) \mapsto(X, Y, Z)=(x+i y, x-i y, z)$ diagonalizing in $\mathbb{C}$ the linear part of (12). In this way system (12) becomes a system in $\mathbb{C}^{3}$ with the form

$$
\begin{aligned}
& \dot{X}=i X+\omega^{2} \mathcal{F}\left(\frac{X+Y}{2}, \frac{i(Y-X)}{2}, Z\right), \\
& \dot{Y}=-i Y+\omega^{2} \mathcal{F}\left(\frac{X+Y}{2}, \frac{i(Y-X)}{2}, Z\right), \\
& \dot{Z}=\mathcal{F}\left(\frac{X+Y}{2}, \frac{i(Y-X)}{2}, Z\right) .
\end{aligned}
$$

It is straightforward to check that this complex system is time-reversible with respect to the linear involution $(X, Y, Z) \mapsto(Y, X, Z)$ that exchanges $X$ and $Y$ if and only if $\mathcal{F}$ is an odd function in second variable, i.e., $\mathcal{F}(x,-y, z)=-\mathcal{F}(x, y, z)$. Notice that this symmetry is inherited by $f_{n}$, hence $f_{n}(x,-y, z)=-f_{n}(x, y, z)$. In this symmetric case both first integrals of the linear part are conserved in a Poincaré-Dulac normal form of (13), see Proposition 10. Hence the complex system admits two independent analytic first integrals $\hat{H}_{1}(X, Y, Z)=X^{2}-Y^{2}+\cdots$ and $\hat{H}_{2}(X, Y, Z)=Z+\cdots$, see Theorem 9, which implies that the real system (12) possesses two independent locally real analytic first integrals $H_{1}(x, y, z)=x^{2}+y^{2}+\cdots$ and $H_{2}(x, y, z)=z+\cdots$. Then the origin of (12) is a 3 -dimensional center and consequently the singularity $\left(x_{0}, 0,0\right)$ of $(2)$ is too.

\section{Proof of Theorem 2}

We take $f_{n}$ independent of $x$ so that in system (2) a decoupling occurs. Thus we deal with the planar subsystem

$$
\dot{y}=z, \dot{z}=f_{n}(y, z)=-\omega^{2} y+\cdots
$$

where the starting terms in $f_{n}(y, z)$ are derived from the assumption of having a zero-Hopf singularity of (2) at the origin, see the beginning of the proof of Theorem 1. By hypothesis (14) has a nondegenerate center at the origin. Therefore the solution $\xi\left(t ;\left(y_{0}, z_{0}\right)\right)=$ $\left(y\left(t ;\left(y_{0}, z_{0}\right)\right), z\left(t ;\left(y_{0}, z_{0}\right)\right)\right)$ of (14) passing at time $t=0$ through the point $\left(y_{0}, z_{0}\right) \in U \subset \mathbb{R}^{2}$ in a sufficiently small neighborhood $U$ of the origin is $T\left(y_{0}, z_{0}\right)$-periodic for any $\left(y_{0}, z_{0}\right) \in U$ and also there is an analytic first integral $H(y, z)=y^{2}+z^{2}+\cdots$ defined in $U$ of (14) . Going back to the full system (2) in $\mathbb{R}^{3}$, it has the solution $\left(x\left(t ;\left(x_{0}, y_{0}, z_{0}\right)\right), \xi\left(t ;\left(y_{0}, z_{0}\right)\right)\right)$ with initial condition $\left(x_{0}, y_{0}, z_{0}\right) \in \mathbb{R} \times U$ and where $x\left(t ;\left(x_{0}, y_{0}, z_{0}\right)\right)=x_{0}+\int_{0}^{t} y\left(t ;\left(y_{0}, z_{0}\right)\right) d t$. We claim that actually the function $x\left(t ;\left(x_{0}, y_{0}, z_{0}\right)\right)$ is also a $T\left(y_{0}, z_{0}\right)$-periodic function implying that the origin of (2) is a 3 -dimensional center. To prove the 
claim we only need to prove that the function $y\left(t ;\left(y_{0}, z_{0}\right)\right)$ has zero average in the interval $\left[0, T\left(y_{0}, z_{0}\right)\right]$. This is true because this average is

$$
\int_{0}^{T\left(y_{0}, y_{0}\right)} y\left(t ;\left(y_{0}, z_{0}\right)\right) d t=\oint_{H=h} d x \equiv 0 \text { for all }\left(y_{0}, z_{0}\right) \in U,
$$

where in the last step we integrate an exact 1-form over closed level curves $\{H(y, z)=h\}$ where $h=H\left(y_{0}, z_{0}\right)$.

Remark 11. We observe Theorem 2 can be restated in terms of the transformed system (12) as follows. If $\frac{\partial \mathcal{F}}{\partial z} \equiv 0$ and the planar vector field $\dot{x}=-y+\omega^{2} \mathcal{F}(x, y), \dot{y}=x$ has a center at the origin in $\mathbb{R}^{2}$ then the origin in $\mathbb{R}^{3}$ is a 3 -dimensional center of $(12)$.

\section{Proof of Theorem 3}

We take $f_{2}(x, y, z)=a_{0}(y, z)+a_{1}(y, z) x+a_{2}(y, z) x^{2}$. A singularity $p_{0}=\left(x_{0}, 0,0\right) \in \mathbb{R}^{3}$ of $(2)$ corresponds to a real solution of the quadratic equation $f_{2}\left(x_{0}, 0,0\right)=0$. It is easy to check that when $a_{2}(0,0)=0$ then either there are no singularities of $(2)$ when $a_{1}(0,0)=0$ or there is just one singularity but without any zero associated eigenvalue otherwise. So we continue the proof assuming that $a_{2}(0,0) \neq 0$. We impose the condition

$$
a_{0}(0,0)=\frac{a_{1}(0,0)^{2}}{4 a_{2}(0,0)}
$$

in order to have a null eigenvalue. Next we have the conditions

$$
\begin{aligned}
\frac{\partial a_{0}}{\partial z}(0,0)= & \frac{a_{1}(0,0)\left[2 a_{2}(0,0) \frac{\partial a_{1}}{\partial z}(0,0)-a_{1}(0,0) \frac{\partial a_{2}}{\partial z}(0,0)\right]}{4 a_{2}^{2}(0,0)} \\
0> & 4 a_{2}^{2}(0,0) \frac{\partial a_{0}}{\partial y}(0,0)-2 a_{1}(0,0) a_{2}(0,0) \frac{\partial a_{1}}{\partial y}(0,0)+ \\
& a_{1}^{2}(0,0) \frac{\partial a_{2}}{\partial y}(0,0)
\end{aligned}
$$

for having also two non-zero pure imaginary eigenvalues.

Since $f_{2}$ is a polynomial of degree 2 we have

$$
\begin{aligned}
& a_{0}(y, z)=b_{0}+b_{1} y+b_{2} z+b_{3} y^{2}+b_{4} y z+b_{5} z^{2}, \\
& a_{1}(y, z)=c_{0}+c_{1} y+c_{2} z, \\
& a_{2}(y, z)=d_{0} .
\end{aligned}
$$

Then $a_{2}(0,0) \neq 0,(15)$ and (16) produces

$$
d_{0} \neq 0, b_{0}=\frac{c_{0}^{2}}{4 d_{0}}, b_{2}=\frac{c_{0} c_{2}}{2 d_{0}}, b_{1}=\frac{2 c_{0} c_{1} d_{0}-K^{2}}{4 d_{0}^{2}}
$$


where in the last condition we have introduced a new parameter $K \neq$ 0 such that inequality (17) reads for $0>-K^{2}$. The coordinates of the zero-Hopf singularity are $p_{0}=\left(-c_{0} /\left(2 d_{0}\right), 0,0\right)$ whose eigenvalues are $\{ \pm i \omega, 0\}$ with $\omega=K /\left(2 d_{0}\right)$. As in the first paragraph of the proof of Theorem 1, we translate $p_{0}$ to the origin; we perform a linear transformation to get the real Jordan canonical form of the linear part of the system and we rescale the time $t \mapsto t / \omega$. Thus we obtain that (2) is transformed into a system (12) of the form

$$
\begin{aligned}
& \dot{x}=-y+\omega^{2} \mathcal{F}(x, y, z) \\
& \dot{y}=x \\
& \dot{z}=\mathcal{F}(x, y, z)
\end{aligned}
$$

with

$$
\begin{aligned}
\mathcal{F}(x, y, z)= & \frac{4 C d_{0}^{2}}{K^{2}} x^{2}+\frac{4 B d_{0}^{2}}{K^{2}} x y+\frac{32 b_{3} d_{0}^{5}}{K^{5}} y^{2}+\frac{4 A d_{0}^{2}}{K^{2}} x z+ \\
& \frac{16 c_{1} d_{0}^{4}}{K^{4}} y z+\frac{8 d_{0}^{4}}{K^{3}} z^{2}
\end{aligned}
$$

after the reparametrization $\left(c_{2}, b_{4}, b_{5}\right) \mapsto(A, B, C)$ with

$$
c_{2}=\frac{16 d_{0}^{4}+A K^{3}}{2 d_{0} K^{2}}, b_{4}=\frac{16 c_{1} d_{0}^{4}+B K^{4}}{4 d_{0}^{2} K^{2}}, b_{5}=\frac{32 d_{0}^{6}+4 A d_{0}^{2} K^{3}+C K^{5}}{2 d_{0} K^{4}} \text {. }
$$

Using the theory developed in [4] and summarized in subsection 2.1 gives that family (18) with $d_{0} \neq 0$ and $K \neq 0$ has not a 3 -dimensional center at the origin because the first associated Melnikov function $\delta_{1}\left(r_{0}, w_{0}\right)$ is

$\delta_{1}\left(r_{0}, w_{0}\right)=\left(A \pi r_{0}^{2} w_{0},-\frac{\pi r_{0}}{K^{5}}\left[-32 b_{3} d_{0}^{5}-4 C d_{0}^{2} K^{3}-16 d_{0}^{4} K^{2} w_{0}^{2}+A K^{5} w_{0}^{2}\right]\right)$

and clearly $\delta_{1}\left(r_{0}, w_{0}\right) \not \equiv 0$ for all possible choice of the parameters. Hence the theorem follows.

\section{Proof of Theorem 4}

If $n=3$ and $f_{3}$ is a cubic homogeneous polynomial, then is a simple exercise to check that there is no zero-Hopf singularity in family (2) if $f_{3}(x, 0,0) \not \equiv 0$. Hence statement (i) is straightforward to check and we concentrate our attention only in proving statement (ii).

Following the notation of the proof of Theorem 3 and taking into account that $f_{3}(x, 0,0) \equiv 0$ we take $f_{3}(x, y, z)=a_{0}(y, z)+a_{1}(y, z) x+$ 
$a_{2}(y, z) x^{2}$ with

$$
\begin{aligned}
& a_{0}(y, z)=b_{6} y^{3}+b_{7} y^{2} z+b_{8} y z^{2}+b_{9} z^{3}, \\
& a_{1}(y, z)=c_{3} y^{2}+c_{4} y z+c_{5} z^{2}, \\
& a_{2}(y, z)=d_{1} y+d_{2} z .
\end{aligned}
$$

Under these conditions it is easy to check that system (2) has zeroHopf singularities only when $d_{2}=0$ and $d_{1}=-K^{2}$ with $K \neq 0$. In short a continuum of singularities $\left(x_{0}, 0,0\right)$ on the $x$-axis appear for any $x_{0} \in \mathbb{R}$ such that all of them except the origin are zero-Hopf points with eigenvalues $\{ \pm i \omega, 0\}$ where $\omega=K x_{0}$.

Pick up one of these zero-Hopf singularities $\left(x_{0}, 0,0\right)$ with $x_{0} \neq 0$ and translate it to the origin by means of $(x, y, z) \mapsto\left(x-x_{0}, y, z\right)$. Next we follow the first paragraph of the proof of Theorem 1 and we do a linear change of variables to obtain the linear part of the system in real Jordan canonical form and also we perform the time rescaling $t \mapsto t / \omega$. After all these transformations system (2) is written as a system (12) of the form

$$
\begin{aligned}
& \dot{x}=-y+\omega^{2} \mathcal{F}(x, y, z) \\
& \dot{y}=x \\
& \dot{z}=\mathcal{F}(x, y, z)
\end{aligned}
$$

where $\mathcal{F}$ is the following cubic polynomial

$$
\begin{aligned}
\mathcal{F}(x, y, z)= & -\frac{c_{5}}{K^{3} x_{0}^{2}} x^{2}-\frac{2+c_{4} x_{0}^{2}}{K^{4} x_{0}^{5}} x y-\frac{c_{3}}{K^{5} x_{0}^{4}} y^{2}+\frac{2}{K^{2} x_{0}^{3}} y z+ \\
& \frac{b_{9} K^{2} x_{0}^{2}-c_{5}}{K^{5} x_{0}^{5}} x^{3}+\frac{-1-c_{4} x_{0}^{2}+b_{8} K^{2} x_{0}^{4}}{K^{6} x_{0}^{8}} x^{2} y- \\
& \frac{c_{3}-b_{7} K^{2} x_{0}^{2}}{K^{7} x_{0}^{7}} x y^{2}+\frac{b_{6}}{K^{6} x_{0}^{6}} y^{3}+\frac{c_{5}}{K^{3} x_{0}^{3}} x^{2} z+\frac{2+c_{4} x_{0}^{2}}{K^{4} x_{0}^{6}} x y z+ \\
& \frac{c_{3}}{K^{5} x_{0}^{5}} y^{2} z-\frac{1}{K^{2} x_{0}^{4}} y z^{2} .
\end{aligned}
$$

Using the theory developed in [4] and summarized in subsection 2.1 gives that the first associated Melnikov functions $\delta_{i}\left(r_{0}, w_{0}\right)$ at the origin for family (19) are the following.

$$
\delta_{1}\left(r_{0}, w_{0}\right)=\left(0,-\frac{\pi r_{0}\left(c_{3}+c_{5} K^{2} x_{0}^{2}\right)}{K^{5} x_{0}^{4}}\right)
$$

and clearly $\delta_{1}\left(r_{0}, w_{0}\right) \equiv 0$ if and only if $c_{3}=-c_{5} K^{2} x_{0}^{2}$. Next we compute

$$
\delta_{2}\left(r_{0}, w_{0}\right)=\left(\frac{\pi r_{0}^{3}\left(b_{7}-4 c_{5}+3 b_{9} K^{2} x_{0}^{2}\right)}{4 K^{3} x_{0}^{3}},-\frac{\pi r_{0}^{2} w_{0}\left(b_{7}-12 c_{5}+3 b_{9} K^{2} x_{0}^{2}\right.}{4 K^{3} x_{0}^{3}}\right)
$$


which vanishes identically only when $c_{5}=0$ and $b_{7}=-3 b_{9} K^{2} x_{0}^{2}$. After we get that

$$
\delta_{3}\left(r_{0}, w_{0}\right)=\left(-\frac{3 b_{9} \pi r_{0}^{4} w_{0}}{2 K x_{0}^{2}}, \frac{b_{9} \pi r_{0}^{3}\left(2+c_{4} x_{0}^{2}+3 K^{4} w_{0}^{2} x_{0}^{4}\right)}{2 K^{5} x_{0}^{6}}\right)
$$

and $\delta_{3}\left(r_{0}, w_{0}\right) \equiv 0$ if and only if $b_{9}=0$. Also we can check that $\delta_{4}\left(r_{0}, w_{0}\right)=\delta_{5}\left(r_{0}, w_{0}\right) \equiv 0$.

The resulting family (2) becomes (3) after the relabeling of parameters $\left(b_{6}, c_{4}, b_{8}\right)=(A, B, C)$. The proof finishes noticing that in (3) we have that $f_{3}(x, y, z)=-K^{2} x^{2} y+A y^{3}+B x y z+C y z^{2}$ is an odd function in the variable $y$ and applying Theorem 1 .

\section{Proof of Theorem 5}

Let $f_{3}(x, y, z)=a_{0}(y, z)+a_{1}(y, z) x+a_{2}(y, z) x^{2}+a_{3} x^{3}$. The singularity $p_{0}=\left(x_{0}, 0,0\right) \in \mathbb{R}^{3}$ of $(2)$ corresponds to a real solution of the cubic equation $f_{3}\left(x_{0}, 0,0\right)=0$. Since we want the origin $(0,0,0)$ to be a singular point of $(2)$ with $n=3$ we must have $a_{0}(0,0)=0$. Then we get $a_{1}(0,0)=0$ to have a null eigenvalue associated to the origin and moreover

$$
\frac{\partial a_{0}}{\partial z}(0,0)=0, \quad \frac{\partial a_{0}}{\partial y}(0,0)<0
$$

for having also two non-zero pure imaginary eigenvalues.

Now we take the following expression of the polynomial $f_{3}$ :

$$
\begin{aligned}
a_{0}(y, z)= & b_{0}+b_{1} y+b_{2} z+b_{3} y^{2}+b_{4} y z+b_{5} z^{2}+b_{6} y^{3}+ \\
& b_{7} y^{2} z+b_{8} y z^{2}+b_{9} z^{3}, \\
a_{1}(y, z)= & c_{0}+c_{1} y+c_{2} z+c_{3} y^{2}+c_{4} y z+c_{5} z^{2}, \\
a_{2}(y, z)= & d_{0}+d_{1} y+d_{2} z, \\
a_{3}= & e_{0} .
\end{aligned}
$$

Conditions $a_{0}(0,0)=a_{1}(0,0)=0$ and (20) yield

$$
b_{0}=c_{0}=b_{2}=0, b_{1}=-K^{2}
$$

with $K \neq 0$. The resulting system (2) with $n=3$ has a singularity at the origin with eigenvalues $\{ \pm i \omega, 0\}$ with $\omega=K$.

As in the first paragraph of the proof of Theorem 1, after performing a linear transformation to get the real Jordan canonical form of the linear part of the system and doing a time rescaling $t \mapsto t / \omega$ we obtain 
that (2) is transformed into a system (12) of the form

$$
\begin{aligned}
& \dot{x}=-y+K^{2} \mathcal{F}(x, y, z) \\
& \dot{y}=x \\
& \dot{z}=\mathcal{F}(x, y, z)
\end{aligned}
$$

with

$$
\begin{aligned}
\mathcal{F}(x, y, z)= & \frac{F}{K^{2}} x^{2}+\frac{b_{3}}{K^{5}} y^{2}+\frac{A}{K^{2}} x z+\frac{c_{1}}{K^{4}} y z+\frac{d_{0}}{K^{3}} z^{2}+\frac{I}{K^{2}} x^{3} \\
& +\frac{C}{K^{2}} x y+\frac{H}{K^{2}} x^{2} y+\frac{E}{K^{2}} x y^{2}+\frac{b_{6}}{K^{6}} y^{3}+\frac{G}{K^{2}} x^{2} z+ \\
& \frac{D}{K^{2}} x y z+\frac{c_{3}}{K^{5}} y^{2} z+\frac{B}{K^{2}} x z^{2}+\frac{d_{1}}{K^{4}} y z^{2}+\frac{e_{0}}{K^{3}} z^{3}
\end{aligned}
$$

after the reparametrization

$$
\left(c_{2}, d_{2}, b_{4}, c_{4}, b_{7}, b_{5}, b_{8}, b_{9}\right) \mapsto(A, B, C, D, E, F, G, H, I)
$$

with

$$
\begin{aligned}
c_{2} & =\frac{2 d_{0}+A K^{3}}{K^{2}}, d_{2}=\frac{3 e_{0}+B K^{3}}{K^{2}}, b_{4}=\frac{c_{1}+C K^{4}}{K^{2}}, \\
c_{4} & =\frac{2 d_{1}+D K^{4}}{K^{2}}, b_{7}=\frac{c_{3}+E K^{5}}{K^{2}}, b_{5}=\frac{d_{0}+A K^{3}+F K^{5}}{K^{4}}, \\
c_{5} & =\frac{3 e_{0}+2 B K^{3}+G K^{5}}{K^{4}}, b_{8}=\frac{d_{1}+D K^{4}+H K^{6}}{K^{4}}, \\
b_{9} & =\frac{e_{0}+B K^{3}+G K^{5}+I K^{7}}{K^{6}} .
\end{aligned}
$$

Now we compute Melnikov functions (as explained in subsection 2.1) for family (21) with $K \neq 0$ in order to detect 3-dimensional centers at the origin. The first associated Melnikov function $\delta_{1}\left(r_{0}, w_{0}\right)$ is

$$
\delta_{1}\left(r_{0}, w_{0}\right)=\left(A \pi r_{0}^{2} w_{0},-\frac{\pi r_{0}}{K^{5}}\left[-b_{3}-F K^{3}+K^{2}\left(-2 d_{0}+A K^{3}\right) w_{0}^{2}\right]\right)
$$

Therefore $\delta_{1}\left(r_{0}, w_{0}\right) \equiv 0$ if and only if

$$
A=d_{0}=0, b_{3}=-F K^{3} .
$$

Under these constrains, the second Melnikov function $\delta_{2}\left(r_{0}, w_{0}\right)=$ $\left(\delta_{2,1}\left(r_{0}, w_{0}\right), \delta_{2,2}\left(r_{0}, w_{0}\right)\right)$ has components

$$
\begin{aligned}
\delta_{2,1}\left(r_{0}, w_{0}\right)= & -\frac{\pi}{4 K^{4}} r_{0}^{3}\left[c_{1} F+E K^{4}+3 I K^{4}+4 B K^{4} w_{0}^{2}\right], \\
\delta_{2,2}\left(r_{0}, w_{0}\right)= & -\frac{\pi}{4 K^{5}} r_{0}^{2} w_{0}\left[-4 c_{3}+5 c_{1} F K-4 G K^{3}+E K^{5}+3 I K^{5}\right. \\
& \left.-8 e_{0} K^{2} w_{0}^{2}+4 B K^{5} w_{0}^{2}\right] .
\end{aligned}
$$


Next it is easy to check that the second Melnikov function $\delta_{2}\left(r_{0}, w_{0}\right)$ vanishes identically if and only if

$$
\begin{aligned}
B & =e_{0}=0, \\
c_{3} & =\frac{1}{4}\left(5 c_{1} F K-4 G K^{3}+E K^{5}+3 I K^{5}\right), \\
E & =-\frac{1}{K^{4}}\left(c_{1} F+3 I K^{4}\right) .
\end{aligned}
$$

Straightforward computations gives the following higher order Melnikov functions. More precisely we get

$$
\delta_{3}\left(r_{0}, w_{0}\right)=\left(-\Delta_{1} \frac{\pi}{4 K^{4}} r_{0}^{4} w_{0}, \frac{\pi}{4 K^{6}} r_{0}^{3}\left[\Delta_{2}-3 K^{2} \Delta_{3} w_{0}^{2}\right]\right)
$$

where $\Delta_{i}$ are polynomials in the parameter space of family (21) whose expressions can be found in the Appendix 1. Also we obtain $\delta_{4}\left(r_{0}, w_{0}\right)=$ $\left(\delta_{4,1}\left(r_{0}, w_{0}\right), \delta_{4,2}\left(r_{0}, w_{0}\right)\right)$ with components

$$
\begin{aligned}
\delta_{4,1}\left(r_{0}, w_{0}\right)= & -\frac{\pi}{48 K^{8}} r_{0}^{5}\left[\Delta_{4}+4 \Delta_{1}\left(3 c_{1}+C K^{4}\right) w_{0}+6 K^{2} \Delta_{5} w_{0}^{2}\right], \\
\delta_{4,2}\left(r_{0}, w_{0}\right)= & \frac{\pi}{48 K^{10}} r_{0}^{4}\left[-4\left(3 c_{1}-4 C K^{4}\right) \Delta_{2}+K^{2} \Delta_{6} w_{0}+\right. \\
& \left.4 K^{2} \Delta_{7} w_{0}^{2}+6 K^{4} \Delta_{8} w_{0}^{3}\right] .
\end{aligned}
$$

The fifth-order Melnikov function $\delta_{5}\left(r_{0}, w_{0}\right)=\left(\delta_{5,1}\left(r_{0}, w_{0}\right), \delta_{5,2}\left(r_{0}, w_{0}\right)\right)$ has components

$$
\begin{aligned}
\delta_{5,1}\left(r_{0}, w_{0}\right)= & -\frac{\pi}{576 K^{12}} r_{0}^{6}\left[12 \Delta_{9}-\Delta_{10} w_{0}+24 K^{2} \Delta_{11} w_{0}^{2}+18 K^{4} \Delta_{12} w_{0}^{3}\right], \\
\delta_{5,2}\left(r_{0}, w_{0}\right)= & \frac{\pi}{48 K^{14}} r_{0}^{5}\left[\Delta_{13}+12 K^{2} \Delta_{14} w_{0}-K^{2} \Delta_{15} w_{0}^{2}+24 K^{4} \Delta_{16} w_{0}^{3}+\right. \\
& \left.18 K^{6} \Delta_{17} w_{0}^{4}\right] .
\end{aligned}
$$

The Bautin ideal $\mathcal{B}=<\Delta_{i}: i \in \mathbb{N}>$ at the origin of (21) is generated by all the Poincaré-Liapunov constants $\Delta_{i}$ and is a polynomial ideal in the polynomial ring $\mathbb{R}[\lambda]$ where

$$
\lambda=\left(K, C, D, F, G, H, I, b_{6}, c_{1}, d_{1}\right) \in \mathbb{R}^{10}
$$

contains some of the parameters of family (21). Recall that several parameters have been fixed, see (22) and (23).

Since $\mathcal{B}$ is Noetherian it is generated by a finite number of polynomials by the Hilbert basis theorem. But unfortunately we do not know this basis a priori. Let $\mathcal{B}_{k}=<\Delta_{1}, \ldots, \Delta_{k}>$ be the ideal generated by the first $k$ Poincaré-Liapunov constants at the origin of (21). Before computing the center variety $\mathbf{V}(\mathcal{B})$ we will do some simplifications. We define $\hat{\mathcal{B}}_{k}=<\hat{\Delta}_{1}, \ldots, \hat{\Delta}_{k}>$ be the ideal generated by some polynomials $\hat{\Delta}_{i}$ that we define sequentially as follows. Let $\hat{\Delta}_{1}=\Delta_{1}$ and 
$\hat{\Delta}_{i} \equiv \Delta_{i} \bmod \hat{\mathcal{B}}_{i-1}$ for $i \geq 2$. Clearly $\hat{\mathcal{B}}_{k}=\mathcal{B}_{k}$. In the former reduction we obtain that $\hat{\Delta}_{7}=\hat{\Delta}_{11}=\hat{\Delta}_{12}=\hat{\Delta}_{16}=\hat{\Delta}_{17}=0$. Relabeling consecutively the subscripts of the $\hat{\Delta}_{i}$ such that we remove the above null polynomials we can work with the ideal $\hat{\mathcal{B}}_{11}=<\hat{\Delta}_{1}, \ldots, \hat{\Delta}_{11}>$. We expect that $\mathbf{V}(\mathcal{B})=\mathbf{V}\left(\hat{\mathcal{B}}_{11}\right)$ where the inclusion $\mathbf{V}(\mathcal{B}) \subset \mathbf{V}\left(\hat{\mathcal{B}}_{11}\right)$ is obvious. To verify the opposite inclusion we will find the minimal irreducible decomposition of the variety $\mathbf{V}\left(\hat{\mathcal{B}}_{11}\right)$. This decomposition will be performed with the help of a computer algebra system, more precisely with the procedure minAssGTZ from the library primdec.lib of the software SiNGULAR using the degree-reverse lexicographic order with $K>C>D>F>G>H>I>b_{6}>c_{1}>d_{1}$ to find the primary decomposition of $\sqrt{\hat{\mathcal{B}}_{11}}$, the radical of $\hat{\mathcal{B}}_{11}$. The output is that $\mathbf{V}\left(\hat{\mathcal{B}}_{11}\right)=\cup_{i=1}^{13} \mathbf{V}\left(J_{i}\right)$ where the irreducible varieties $\mathbf{V}\left(J_{i}\right)$ are the varieties associated to the ideals $J_{i}$ listed in Appendix 2.

Finally we must verify the each point of the varieties $\mathbf{V}\left(J_{i}\right)$ corresponds with a system (21) having a 3-dimensional center at the origin. We shall see that sometimes this is not true and we need to compute higher order Melnikov functions $\delta_{j}\left(r_{0}, w_{0}\right)$ with $j \geq 6$ at the origin of family (21) restricted to $\lambda \in \mathbf{V}\left(J_{i}\right)$.

Now we recall that in family $(21)$ the parameter $K \neq 0$ and therefore we do not take into account any of the varieties $\mathbf{V}\left(J_{i}\right)$ with $i \in$ $\{2,11,12\}$ because they have the polynomial $K$ as generator.

7.1. The variety $\mathbf{V}\left(J_{1}\right)$. Since the variety $\mathbf{V}\left(J_{1}\right)$ is defined by

$$
\mathbf{V}\left(J_{1}\right)=\left\{\lambda \in \mathbb{R}^{10}: d_{1}=c_{1}=b_{6}=I=H=D F-C G=0\right\}
$$

we get the associated family (21) with

$$
K^{2} \mathcal{F}(x, y, z)=F x^{2}+C x y-F y^{2}+G x^{2} z+D x y z-G y^{2} z
$$

and the condition $D F-C G=0$.

By Theorem 1 and Remark 11 we have that if $F=G=0$ or $D=$ $G=0$ then (21) has a 3 -dimensional center at the origin.

Therefore only remains the study of the case $G \neq 0$. In this case we can put $C=D F / G$ and after rescaling the time by $t \mapsto G t$ system (21) becomes

$$
\begin{aligned}
& \dot{x}=-G y+K^{2} \hat{\mathcal{F}}(x, y, z) \\
& \dot{y}=G x \\
& \dot{z}=\hat{\mathcal{F}}(x, y, z),
\end{aligned}
$$

with $K^{2} \hat{\mathcal{F}}(x, y, z)=\left(G x^{2}+D x y-G y^{2}\right)(F+G z)$. Performing the planar rotation in the $(x, y)$-plane of angle $\theta^{*}=-\frac{1}{2} \operatorname{arccot}(D /(2 G))$ 
system (25) is greatly simplified because $2 \cos \left(2 \theta^{*}\right)+\left(D \sin \left(2 \theta^{*}\right) / G=0\right.$. Indeed, doing the change of variables

$$
\left(\begin{array}{l}
u \\
v \\
w
\end{array}\right)=\left(\begin{array}{ccc}
\cos \theta^{*} & -\sin \theta^{*} & 0 \\
\sin \theta^{*} & \cos \theta^{*} & 0 \\
0 & 0 & 1
\end{array}\right)\left(\begin{array}{l}
x \\
y \\
z
\end{array}\right)
$$

system (25) becomes

$$
\begin{aligned}
& \dot{u}=-v+C_{1} \mathcal{G}(u, v, w), \\
& \dot{v}=u+C_{2} \mathcal{G}(u, v, w), \\
& \dot{w}=C_{3} \mathcal{G}(u, v, w),
\end{aligned}
$$

where $\mathcal{G}(u, v, w)=u v(F+G w)$ and $C_{i}$ are certain constants depending on the parameters of the family. Removing common factors we see that two planar subsystems are associated to (27), namely

$$
\dot{u}=-1+C_{1} u(F+G w), \dot{w}=C_{3} u(F+G w),
$$

and

$$
\dot{v}=1+C_{2} v(F+G w), \dot{w}=C_{3} v(F+G w) .
$$

Since the origin is a regular point of both (28) and (29) there are around it analytic first integrals $\hat{H}_{1}(u, w)$ of $(28)$ and $\hat{H}_{2}(v, w)$ of $(29)$. Additionally both functions $\hat{H}_{1}(u, w)$ and $\hat{H}_{2}(v, w)$ are first integrals of the full system $(27)$. Let $\nabla=\left(\partial_{u}, \partial_{v}, \partial_{w}\right)$ be the gradient operator. Since the gradient vectors $\nabla \hat{H}_{1}(u, w)=\left(\partial_{u} \hat{H}_{1}, 0, \partial_{w} \hat{H}_{1}\right)$ and $\nabla \hat{H}_{2}(v, w)=\left(0, \partial_{v} \hat{H}_{2}, \partial_{w} \hat{H}_{2}\right)$ are linearly independent except in the zero Lebesgue measure set $\left\{(u, v, w): \partial_{u} \hat{H}_{1}=\partial_{v} \hat{H}_{2}=0\right\}$ we conclude that $\hat{H}_{1}(u, w)$ and $\hat{H}_{2}(v, w)$ are two functionally independent analytical first integrals of (27) almost everywhere in a neighborhood of the origin, that is in a full Lebesgue measure (dense) neighborhood of the origin. In short, going back through the linear change (26) one has that the pull back $H_{1}(x, y, z)=\hat{H}_{1}\left(\cos \theta^{*} x-\sin \theta^{*} y, z\right)$ and $H_{2}(x, y, z)=\hat{H}_{2}\left(\sin \theta^{*} x+\cos \theta^{*} y, z\right)$ also are functionally independent analytical first integrals of family $(21)$ with $\mathcal{F}$ given by (24). Taking into account that the linear part of (21) there is no restriction in assuming that $H_{1}(x, y, z)=x^{2}+y^{2}+\cdots$ and $H_{2}(x, y, z)=z+\cdots$ and therefore family (21) with $\mathcal{F}$ given by (24) has a center at the origin. This proves statement (i) of Theorem 5.

7.2. The variety $\mathbf{V}\left(J_{3}\right)$. Taking into account the expressions of the generators of the ideal $J_{3}$ we see that the variety $\mathbf{V}\left(J_{3}\right)$ is given by

$$
\mathbf{V}\left(J_{3}\right)=\left\{\lambda \in \mathbb{R}^{10}: d_{1}=c_{1}=H=I=G=D=b_{6}=0\right\}
$$

and therefore $\mathbf{V}\left(J_{3}\right) \subset \mathbf{V}\left(J_{1}\right)$. 
7.3. The variety $\mathbf{V}\left(J_{4}\right)$. From the generators of the ideal $J_{4}$ we get that

$$
\begin{aligned}
\mathbf{V}\left(J_{4}\right)= & \left\{\lambda \in \mathbb{R}^{10}: d_{1}=c_{1}=F=0, G=-2 K^{2} I,\right. \\
& \left.D=-\frac{4}{3} K^{2} H, b_{6}=\frac{1}{4} K^{2} D\right\} .
\end{aligned}
$$

Imposing that $\lambda \in \mathbf{V}\left(J_{4}\right)$ we can compute the sixth-order Melnikov function at the origin of $(21)$

$$
\delta_{6}\left(r_{0}, w_{0}\right)=I\left(H^{2}+9 I^{2}\right) \pi\left(\frac{5}{864} r_{0}^{7}, \frac{7}{288} r_{0}^{6} w_{0}\right)
$$

from where we see that $\delta_{6}\left(r_{0}, w_{0}\right) \equiv 0$ if and only $I=0$. Therefore its associated family (21) has

$$
K^{2} \mathcal{F}(x, y, z)=C x y+H x^{2} y-\frac{H}{3} y^{3}-\frac{4}{3} H K^{2} x y z,
$$

and by Theorem 1 family (21) has a 3-dimensional center at the origin. This proves statement (ii) of Theorem 5 .

7.4. The variety $\mathbf{V}\left(J_{5}\right)$. From the generators of the ideal $J_{5}$ one has that

$$
\begin{gathered}
\mathbf{V}\left(J_{5}\right)=\left\{\lambda \in \mathbb{R}^{10}: d_{1}=c_{1}=0, G=-\frac{3}{2} K^{2} I, D=-K^{2} H,\right. \\
\left.b_{6}=\frac{1}{3} K^{2} D, 2 F H-3 C I=0\right\}
\end{gathered}
$$

hence we get the associated family (21) with

$$
\begin{aligned}
K^{2} \mathcal{F}(x, y, z)= & F x^{2}+C x y-F y^{2}+I x^{3}+H x^{2} y-3 I x y^{2}-\frac{H}{3} y^{3}- \\
& \frac{3}{2} I K^{2} x^{2} z-H K^{2} x y z+\frac{3}{2} I K^{2} y^{2} z,
\end{aligned}
$$

and the condition $2 F H-3 C I=0$. Then statement (viii) of Theorem 5 follows.

By Theorem 1 and Remark 11 we have that if $F=I=0$ or $H=$ $I=0$ then (21) has a 3 -dimensional center at the origin.

Hence only remains the study of $I \neq 0$ and we take $C=2 F H /(3 I)$. Doing the $z$-translation $(x, y, z) \mapsto(x, y, z-\alpha)$ with $\alpha=2 F /\left(3 I K^{2}\right)$ we obtain the same system (21) with $K^{2} \mathcal{F}(x, y, z)$ given by (31) but with $F=C=0$. Under these new constrains one has $\delta_{i}\left(r_{0}, w_{0}\right) \equiv 0$ for all $i \leq 11$. This proves Remark 6 . 
7.5. The variety $\mathbf{V}\left(J_{6}\right)$. From the generators of the ideal $J_{6}$ we get

$$
\begin{aligned}
\mathbf{V}\left(J_{5}\right)= & \left\{\lambda \in \mathbb{R}^{10}: d_{1}=c_{1}=F=C=0, b_{6}=-\frac{1}{3} K^{4} H,\right. \\
& 2 G H-3 D I=0\} .
\end{aligned}
$$

If $\lambda \in \mathbf{V}\left(J_{6}\right)$ we known that the first Melnikov function $\delta_{i}\left(r_{0}, w_{0}\right) \equiv 0$ for $i=1, \ldots, 5$ but $\delta_{6}\left(r_{0}, w_{0}\right) \not \equiv 0$. After some involved computations we can see that $\delta_{6}\left(r_{0}, w_{0}\right) \equiv 0$ if and only if the following polynomials in $\mathbb{R}[\lambda]$

$$
\begin{aligned}
\Lambda_{1}= & 8 D G H-6 D^{2} I+24 G^{2} I+10 G H^{2} K^{2}-6 D H I K^{2}+ \\
& 54 G I^{2} K^{2}+3 H^{2} I K^{4}+27 I^{3} K^{4} \\
\Lambda_{2}= & 32 D G H-30 D^{2} I+72 G^{2} I+66 G H^{2} K^{2}-78 D H I K^{2}+ \\
& 126 G I^{2} K^{2}+3 H^{2} I K^{4}+27 I^{3} K^{4}
\end{aligned}
$$

vanishes. If we define $\Lambda_{3}=2 G H-3 D I$, one of the generators of the ideal $J_{5}$, and compute resultants between the $\Lambda_{i}$ with respect to $G$ yields

$$
\begin{aligned}
& \mathcal{R}\left[\Lambda_{1}, \Lambda_{3}, G\right]=12 I\left(H^{2}+9 I^{2}\right)\left(D+H K^{2}\right)\left(2 D+H K^{2}\right), \\
& \mathcal{R}\left[\Lambda_{2}, \Lambda_{3}, G\right]=12 I\left(H^{2}+9 I^{2}\right)\left(D+H K^{2}\right)\left(6 D+H K^{2}\right) .
\end{aligned}
$$

Therefore two cases arise to annul the above resultants:

(a) Put the parameter $I=0$. Then the associated family (21) has

$$
K^{2} \mathcal{F}(x, y, z)=H x^{2} y-\frac{H}{3} y^{3}+G x^{2} z+D x y z-G y^{2} z,
$$

with the condition $G H=0$. Clearly if $G=0$ then by Theorem 1 family (21) has a 3 -dimensional center at the origin, hence we can assume that $H=0$ and $G \neq 0$. In this last case, family (21) has $\mathcal{F}(x, y, z)$ as in (24) (with the particular election of parameters $F=C=0$ ) and we have already proved that family $(21)$ has a 3 -dimensional center at the origin. Then statement (iii) of Theorem 5 is proved.

(b) Let $D=-H K^{2}$ and $I \neq 0$. Then $\delta_{i}\left(r_{0}, w_{0}\right) \equiv 0$ for $i=6,7,8$ if and only if $G=-3 I K^{2} / 2$, in which case family (21) has

$$
\begin{aligned}
K^{2} \mathcal{F}(x, y, z)= & I x^{3}+H x^{2} y-3 I x y^{2}-\frac{H}{3} y^{3}-\frac{3}{2} I K^{2} x^{2} z-H K^{2} x y z+ \\
& \frac{3}{2} I K^{2} y^{2} z
\end{aligned}
$$

with $I \neq 0$. In this case, the associated family (21) has $\mathcal{F}(x, y, z)$ as in (31) (with the parameters $F=C=0$ ) and we have already proved that in this case family (21) has a 3 -dimensional center at the origin. 
7.6. The variety $\mathbf{V}\left(J_{7}\right)$. From the generators of the ideal $J_{7}$ we obtain that

$$
\mathbf{V}\left(J_{5}\right)=\left\{\lambda \in \mathbb{R}^{10}: d_{1}=c_{1}=H=I=b_{6}=C=F=D=G=0\right\}
$$

giving rise to the trivial linear system $\dot{x}=-y, \dot{y}=x, \dot{z}=0$.

7.7. The variety $\mathbf{V}\left(J_{8}\right)$. From the generators of the ideal $J_{8}$ we see that in order to express the variety $\mathbf{V}\left(J_{8}\right)$ we must impose the following parameter constraints

$$
d_{1}=c_{1}=0, G=-K^{2} I, b_{6}=K^{4} H+2 K^{2} D
$$

but additional polynomial restrictions are needed to determine the variety $\mathbf{V}\left(J_{8}\right)$. Then we will split the problem in several subcases.

Fist we assume that $I=F=H=0$. Then we obtain also $D=0$ and $\lambda \in \mathbf{V}\left(J_{8}\right)$ but the resulting associated family (21) is a particular case of system (24).

Assume now that $I=F=0$ but $H \neq 0$ and $D=-H K^{2}$. Then $\lambda \in \mathbf{V}\left(J_{8}\right)$ and the associated family (21) has

$$
K^{2} \mathcal{F}(x, y, z)=C x y+H x^{2} y-H y^{3}-H K^{2} x y z,
$$

with $H \neq 0$. By Theorem 1 family (21) has a 3 -dimensional center at the origin. This proves statement (iv) of Theorem 5 .

We can suppose now that $I=F=0, H \neq 0$ and $D \neq-H K^{2}$. Then we necessarily get $D=-H K^{2} / 2$ to have $\lambda \in \mathbf{V}\left(J_{8}\right)$. In this case family (21) has

$$
K^{2} \mathcal{F}(x, y, z)=C x y+H x^{2} y-\frac{1}{2} H K^{2} x y z,
$$

with $H \neq 0$. By Theorem 1 family (21) has a 3-dimensional center at the origin and statement $(\mathrm{v})$ of Theorem 5 is proved.

Let $I=0$ but $F \neq 0$. If $D \neq 0$ then $\lambda \notin \mathbf{V}\left(J_{8}\right)$, so we assume that $D=0$. Then $\lambda \in \mathbf{V}\left(J_{8}\right)$ if and only if $H=0$. Hence family (21) becomes a particular case of system (24).

Finally we deal with the case $I \neq 0$. Then we have $C=-D F /\left(I K^{2}\right)$ and $\lambda \in \mathbf{V}\left(J_{8}\right)$ if and only if $2 D^{2}+3 D H K^{2}+H^{2} K^{4}-I^{2} K^{4}=0$. We also check that under this constrained one has $\delta_{i}\left(r_{0}, w_{0}\right) \equiv 0$ for $i=1, \ldots, 8$. The resulting family (21) has

$$
\begin{aligned}
K^{2} \mathcal{F}(x, y, z)= & F x^{2}-\frac{D F}{I K^{2}} x y-F y^{2}+I x^{3}+H x^{2} y-3 I x y^{2}+ \\
& \left(\frac{2 D}{K^{2}}+H\right) y^{3}-I K^{2} x^{2} z+D x y z+I K^{2} y^{2} z
\end{aligned}
$$

with the conditions $I \neq 0$ and $2 D^{2}+3 D H K^{2}+H^{2} K^{4}-I^{2} K^{4}=0$. Then statement (ix) of Theorem 5 is proved. Notice that Theorem 1 
and Remark 11 do not work in this case because $I \neq 0$. Also we have seen that, in the special case $F=0$ then $\delta_{i}\left(r_{0}, w_{0}\right) \equiv 0$ for $i=1, \ldots, 11$.

7.8. The variety $\mathbf{V}\left(J_{9}\right)$. From the generators of the ideal $J_{9}$ we see that

$$
\begin{aligned}
\mathbf{V}\left(J_{9}\right)= & \left\{\lambda \in \mathbb{R}^{10}: d_{1}=c_{1}=F=C=0, G=-K^{2} I,\right. \\
& \left.b_{6}=K^{4} H+2 K^{2} D\right\}
\end{aligned}
$$

We get that either $I \neq 0$ and then the resulting family (21) becomes a particular case with $F=0$ of (36) or $I=0$ and therefore the associated family (21) has

$$
K^{2} \mathcal{F}(x, y, z)=\left(\frac{2 D}{K^{2}}+H\right) y^{3}+H x^{2} y+D x y z .
$$

Using Theorem 1 we obtain that (21) has a 3-dimensional center at the origin proving statement (vi) of Theorem 5 .

7.9. The variety $\mathbf{V}\left(J_{10}\right)$. The generators of the ideal $J_{10}$ yield

$$
\mathbf{V}\left(J_{10}\right)=\left\{\lambda \in \mathbb{R}^{10}: d_{1}=b_{6}=I=G=0, D=-K^{2} H\right\} .
$$

Hence the associated family (21) has

$$
\begin{aligned}
K^{2} \mathcal{F}(x, y, z)= & F x^{2}+C x y-F y^{2}+H x^{2} y-\frac{c_{1} F}{K^{4}} x y^{2}+\frac{c_{1}}{K^{2}} y z- \\
& H K^{2} x y z+\frac{c_{1} F}{K^{2}} y^{2} z .
\end{aligned}
$$

Then statement (x) of Theorem 5 is proved changing the name $c_{1} \mapsto A$. Further computations reveal that $\delta_{i}\left(r_{0}, w_{0}\right) \equiv 0$ for $i=1, \ldots, 10$. We notice that by Theorem 1 and Remark 11 we have that if $F=0$ or $c_{1}=H=0$ then (21) has a 3 -dimensional center at the origin proving thus the second part of Remark 6.

7.10. The variety $\mathbf{V}\left(J_{13}\right)$. The generators of the ideal $J_{13}$ yield

$$
\mathbf{V}\left(J_{13}\right)=\left\{\lambda \in \mathbb{R}^{10}: I=G=F=0\right\} .
$$

Hence the associated family (21) has

$$
\begin{aligned}
K^{2} \mathcal{F}(x, y, z)= & C x y+\frac{c_{1}}{K^{2}} y z+H x^{2} y+\frac{b_{6}}{K^{4}} y^{3}+D x y z+ \\
& \frac{d_{1}}{K^{2}} y z^{2} .
\end{aligned}
$$

By Theorem 1 family (21) has a 3-dimensional center at the origin. This proves statement (vii) of Theorem 5 after changing the name of parameters $\left(c_{1}, b_{6}, d_{1}\right) \mapsto(A, B, E)$. 


\section{Appendix 1}

$$
\begin{aligned}
& \Delta_{1}=-2 d_{1} F+c_{1} G+3 c_{1} I K^{2}, \\
& \Delta_{2}=-b_{6} F+D F K^{2}-C G K^{2}+F H K^{4}-2 C I K^{4} \text {, } \\
& \Delta_{3}=2 d_{1} F+c_{1} G-c_{1} I K^{2}, \\
& \Delta_{4}=-5 b_{6} c_{1} F-2 c_{1}^{2} I-6 C d_{1} F K^{2}+3 c_{1} D F K^{2}+16 b_{6} G K^{2}+ \\
& 2 c_{1} C G K^{2}+2 b_{6} C F K^{4}+3 c_{1} F H K^{4}+18 b_{6} I K^{4}+3 c_{1} C I K^{4}- \\
& 2 C D F K^{6}+2 C^{2} G K^{6}+8 G H K^{6}-4 D I K^{6}-2 C F H K^{8}+ \\
& 4 C^{2} I K^{8}+6 H I K^{8} \text {, } \\
& \Delta_{5}=-13 c_{1} d_{1} F-4 c_{1}^{2} G+9 c_{1}^{2} I K^{2}+2 C d_{1} F K^{4}+2 c_{1} C G K^{4}+6 d_{1} I K^{4} \text {, } \\
& \Delta_{6}=-47 b_{6} c_{1} F-14 c_{1}^{2} I-42 C d_{1} F K^{2}+33 c_{1} D F K^{2}+4 b_{6} G K^{2}- \\
& 52 c_{1} C G K^{2}+2 b_{6} C F K^{4}+33 c_{1} F H K^{4}+18 b_{6} I K^{4}- \\
& 57 c_{1} C I K^{4}-2 C D F K^{6}+2 C^{2} G K^{6}+20 G H K^{6}-28 D I K^{6}- \\
& 2 C F H K^{8}+4 C^{2} I K^{8}+6 H I K^{8} \text {, } \\
& \Delta_{7}=-24 c_{1} d_{1} F-6 c_{1}^{2} G+18 c_{1}^{2} I K^{2}-10 C d_{1} F K^{4}-7 c_{1} C G K^{4}+ \\
& 3 c_{1} C I K^{6} \text {, } \\
& \Delta_{8}=-33 c_{1} d_{1} F-24 c_{1}^{2} G-8 d_{1} G K^{2}+9 c_{1}^{2} I K^{2}+2 C d_{1} F K^{4}+ \\
& 2 c_{1} C G K^{4}+6 d_{1} I K^{4} \\
& \Delta_{9}=4 b_{6} c_{1}^{2} F-16 b_{6} d_{1} F K^{2}-8 c_{1} C d_{1} F K^{2}-4 c_{1}^{2} D F K^{2}+8 b_{6} c_{1} G K^{2}+ \\
& 8 c_{1}^{2} C G K^{2}-9 b_{6} c_{1} C F K^{4}-4 c_{1}^{2} F H K^{4}+24 b_{6} c_{1} I K^{4}+ \\
& 18 c_{1}^{2} C I K^{4}-6 C^{2} d_{1} F K^{6}-4 b_{6} D F K^{6}+7 c_{1} C D F K^{6}- \\
& 8 d_{1} F^{3} K^{6}+16 b_{6} C G K^{6}-2 c_{1} C^{2} G K^{6}+4 c_{1} F^{2} G K^{6}- \\
& 8 d_{1} F H K^{6}+4 c_{1} G H K^{6}+2 b_{6} C^{2} F K^{8}+4 D^{2} F K^{8}-4 C D G K^{8}+ \\
& 7 c_{1} C F H K^{8}+18 b_{6} C I K^{8}-5 c_{1} C^{2} I K^{8}+12 c_{1} F^{2} I K^{8}+ \\
& 12 c_{1} H I K^{8}-2 C^{2} D F K^{10}+2 C^{3} G K^{10}+4 D F H K^{10}+ \\
& 8 C G H K^{10}-12 C D I K^{10}-2 C^{2} F H K^{12}+4 C^{3} I K^{12}+ \\
& 6 C H I K^{12} \text {, } \\
& \Delta_{10}=168 c_{1}^{2} d_{1} F-84 c_{1}^{3} G+294 b_{6} c_{1}^{2} F K^{2}-108 c_{1}^{3} I K^{2}+ \\
& 540 b_{6} d_{1} F K^{4}+810 c_{1} C d_{1} F K^{4}-150 c_{1}^{2} D F K^{4}-246 b_{6} c_{1} G K^{4}+ \\
& 159 c_{1}^{2} C G K^{4}-84 b_{6} c_{1} C F K^{6}+48 d_{1} D F K^{6}+24 C d_{1} G K^{6}- \\
& 36 c_{1} D G K^{6}-150 c_{1}^{2} F H K^{6}-810 b_{6} c_{1} I K^{6}-381 c_{1}^{2} C I K^{6}+ \\
& 12 C^{2} d_{1} F K^{8}-24 b_{6} D F K^{8}+60 c_{1} C D F K^{8}+208 d_{1} F^{3} K^{8}- \\
& 24 b_{6} C G K^{8}-150 c_{1} C^{2} G K^{8}+40 c_{1} F^{2} G K^{8}+84 d_{1} F H K^{8}- \\
& 210 c_{1} G H K^{8}-72 C d_{1} I K^{8}+84 c_{1} D I K^{8}+24 D^{2} F K^{10}- \\
& 24 C D G K^{10}+60 c_{1} C F H K^{10}-246 c_{1} C^{2} I K^{10}- \\
& 168 c_{1} F^{2} I K^{10}-270 c_{1} H I K^{10}+24 D F H K^{12}+ \\
& 24 C G H K^{12}-96 C D I K^{12} \text {, }
\end{aligned}
$$




$$
\begin{aligned}
& \Delta_{11}=-78 c_{1}^{2} d_{1} F-12 c_{1}^{3} G-12 d_{1}^{2} F K^{2}+6 c_{1} d_{1} G K^{2}+66 c_{1}^{3} I K^{2}- \\
& 13 c_{1} C d_{1} F K^{4}+2 c_{1}^{2} C G K^{4}+54 c_{1} d_{1} I K^{4}-12 d_{1} D F K^{6}- \\
& 6 c_{1} D G K^{6}+15 c_{1}^{2} C I K^{6}+2 C^{2} d_{1} F K^{8}+2 c_{1} C^{2} G K^{8}+ \\
& 6 C d_{1} I K^{8}+6 c_{1} D I K^{8} \\
& \Delta_{12}=-120 c_{1}^{2} d_{1} F-75 c_{1}^{3} G-64 d_{1}^{2} F K^{2}-64 c_{1} d_{1} G K^{2}+45 c_{1}^{3} I K^{2}+ \\
& 20 c_{1} C d_{1} F K^{4}+20 c_{1}^{2} C G K^{4}+72 c_{1} d_{1} I K^{4}+8 d_{1} D F K^{6}+ \\
& 8 C d_{1} G K^{6}+8 c_{1} D G K^{6} \text {, } \\
& \Delta_{13}=84 b_{6} c_{1}^{2} F-24 c_{1}^{3} I+240 b_{6} d_{1} F K^{2}+48 c_{1} C d_{1} F K^{2}- \\
& 108 c_{1}^{2} D F K^{2}+72 b_{6} c_{1} G K^{2}+108 c_{1}^{2} C G K^{2}+378 b_{6}^{2} F K^{4}- \\
& 63 b_{6} c_{1} C F K^{4}-108 c_{1}^{2} F H K^{4}-72 b_{6} c_{1} I K^{4}+204 c_{1}^{2} C I K^{4}+ \\
& 90 C^{2} d_{1} F K^{6}-366 b_{6} D F K^{6}+123 c_{1} C D F K^{6}+72 d_{1} F^{3} K^{6}+ \\
& 294 b_{6} C G K^{6}-93 c_{1} C^{2} G K^{6}-24 c_{1} F^{2} G K^{6}+48 d_{1} F H K^{6}+ \\
& 72 c_{1} G H K^{6}+48 C d_{1} I K^{6}-72 c_{1} D I K^{6}+210 b_{6} C^{2} F K^{8}+ \\
& 36 D^{2} F K^{8}+200 b_{6} F^{3} K^{8}-36 C D G K^{8}-132 b_{6} F H K^{8}+ \\
& 123 c_{1} C F H K^{8}+612 b_{6} C I K^{8}-246 c_{1} C^{2} I K^{8}-96 c_{1} F^{2} I K^{8}- \\
& 24 c_{1} H I K^{8}-210 C^{2} D F K^{10}-200 D F^{3} K^{10}+210 C^{3} G K^{10}+ \\
& 200 C F^{2} G K^{10}-162 D F H K^{10}+90 C G H K^{10}+48 C D I K^{10}- \\
& 96 F G I K^{10}-210 C^{2} F H K^{12}-200 F^{3} H K^{12}-198 F H^{2} K^{12}+ \\
& 420 C^{3} I K^{12}+400 C F^{2} I K^{12}+348 C H I K^{12}-144 F I^{2} K^{12} \text {, } \\
& \Delta_{14}=39 b_{6} c_{1}^{2} F-2 c_{1}^{3} I-104 b_{6} d_{1} F K^{2}-70 c_{1} C d_{1} F K^{2}-41 c_{1}^{2} D F K^{2}- \\
& 16 b_{6} c_{1} G K^{2}+30 c_{1}^{2} C G K^{2}-79 b_{6} c_{1} C F K^{4}-24 d_{1} D F K^{4}+ \\
& 24 C d_{1} G K^{4}-41 c_{1}^{2} F H K^{4}+114 b_{6} c_{1} I K^{4}+121 c_{1}^{2} C I K^{4}- \\
& 54 C^{2} d_{1} F K^{6}-20 b_{6} D F K^{6}+61 c_{1} C D F K^{6}-64 d_{1} F^{3} K^{6}- \\
& 88 c_{1} C^{2} G K^{6}-16 c_{1} F^{2} G K^{6}-88 d_{1} F H K^{6}-8 c_{1} G H K^{6}+ \\
& 48 C d_{1} I K^{6}-4 c_{1} D I K^{6}+2 b_{6} C^{2} F K^{8}+20 D^{2} F K^{8}- \\
& 20 C D G K^{8}+61 c_{1} C F H K^{8}+18 b_{6} C I K^{8}-113 c_{1} C^{2} I K^{8}+ \\
& 48 c_{1} F^{2} I K^{8}+54 c_{1} H I K^{8}-2 C^{2} D F K^{10}+2 C^{3} G K^{10}+ \\
& 20 D F H K^{10}+24 C G H K^{10}-76 C D I K^{10}-2 C^{2} F H K^{12}+ \\
& 4 C^{3} I K^{12}+6 C H I K^{12} \text {, }
\end{aligned}
$$




$$
\begin{aligned}
\Delta_{15}= & 816 c_{1}^{2} d_{1} F+132 c_{1}^{3} G+1428 b_{6} c_{1}^{2} F K^{2}-288 d_{1}^{2} F K^{2}+ \\
& 144 c_{1} d_{1} G K^{2}-36 c_{1}^{3} I K^{2}+2304 b_{6} d_{1} F K^{4}+3648 c_{1} C d_{1} F K^{4}- \\
& 780 c_{1}^{2} D F K^{4}+1518 b_{6} c_{1} G K^{4}+2979 c_{1}^{2} C G K^{4}+432 c_{1} d_{1} I K^{4}- \\
& 84 b_{6} c_{1} C F K^{6}-96 d_{1} D F K^{6}+888 C d_{1} G K^{6}+36 c_{1} D G K^{6}- \\
& 780 c_{1}^{2} F H K^{6}-810 b_{6} c_{1} I K^{6}+735 c_{1}^{2} C I K^{6}+312 C^{2} d_{1} F K^{8}- \\
& 24 b_{6} D F K^{8}+60 c_{1} C D F K^{8}+832 d_{1} F^{3} K^{8}-24 b_{6} C G K^{8}+ \\
& 150 c_{1} C^{2} G K^{8}+664 c_{1} F^{2} G K^{8}+192 d_{1} F H K^{8}-102 c_{1} G H K^{8}+ \\
& 648 C d_{1} I K^{8}+804 c_{1} D I K^{8}+24 D^{2} F K^{10}-24 C D G K^{10}+ \\
& 60 c_{1} C F K^{10}-246 c_{1} C^{2} I K^{10}-168 c_{1} F^{2} I K^{10}- \\
& 270 c_{1} H I K^{10}+24 D F H K^{12}+24 C G H K^{12}-96 C D I K^{12}, \\
\Delta_{16}= & -233 c_{1}^{2} d_{1} F-122 c_{1}^{3} G-24 d_{1}^{2} F K^{2}-36 c_{1} d_{1} G K^{2}+ \\
& 111 c_{1}^{3} I K^{2}-63 c_{1} C d_{1} F K^{4}-48 c_{1}^{2} C G K^{4}+90 c_{1} d_{1} I K^{4}- \\
& 28 d_{1} D F K^{6}-16 C d_{1} G K^{6}-22 c_{1} D G K^{6}+15 c_{1}^{2} C I K^{6}+ \\
& 2 C^{2} d_{1} F K^{8}+2 c_{1} C^{2} G K^{8}+6 C d_{1} I K^{8}+6 c_{1} D I K^{8}, \\
= & -260 c_{1}^{2} d_{1} F-215 c_{1}^{3} G-144 d_{1}^{2} F K^{2}-224 c_{1} d_{1} G K^{2}+ \\
& 45 c_{1}^{3} I K^{2}+20 c_{1} C d_{1} F K^{4}+20 c_{1}^{2} C G K^{4}+72 c_{1} d_{1} I K^{4}+ \\
& 8 d_{1} D F K^{6}+8 C d_{1} G K^{6}+8 c_{1} D G K^{6} .
\end{aligned}
$$


9. Appendix 2

$$
\begin{aligned}
J_{1}= & <d_{1}, c_{1}, b_{6}, I, H, D F-C G>, \\
J_{2}= & <d_{1}, c_{1}, b_{6}, K> \\
J_{3}= & <d_{1}, c_{1}, H^{2}+9 I^{2}, 2 G H-3 D I, \\
& D H+6 G I, 3 G^{2}-4 H b_{6}, D G+8 I b_{6}, \\
& 3 D^{2}+16 H b_{6}, 2 K^{2} I+G, 4 K^{2} H+3 D, \\
& 6 K^{2} D-4 b_{6}>, \\
J_{4}= & <d_{1}, c_{1}, F, 2 G H-3 D I, \\
& D G+8 I b_{6}, 3 D^{2}+16 H b_{6}, 2 K^{2} I+G, \\
& 4 K^{2} H+3 D, K^{2} D-4 b_{6}>, \\
J_{5}= & <d_{1}, c_{1}, 2 G H-3 D I, 2 F H-3 C I, \\
& 2 D G+9 I b_{6}, D F-C G, D^{2}+3 H b_{6}, \\
& 3 K^{2} I+2 G, K^{2} H+D, 2 C G^{2}+9 F I b_{6}, \\
& K^{2} D-3 b_{6}, K^{2} C G-3 F b_{6}>, \\
J_{6}= & <d_{1}, c_{1}, F, C, 2 G H-3 D I, \\
& K^{4} H+3 b_{6}, K^{4} D I+2 G b_{6}>, \\
J_{7}= & <d_{1}, c_{1}, H^{2}+9 I^{2}, 2 G H-3 D I, \\
& 2 F H-3 C I, D H+6 G I, C H+6 F I, \\
& D F-C G, D^{2}+4 G^{2}, C D+4 F G, \\
& C^{2}+4 F^{2}, K^{4} H+3 b_{6}, 3 K^{4} I^{2}-H b_{6}, \\
& 2 K^{4} G I-D b_{6}, 2 K^{4} F I-C b_{6}, \\
& K^{4} D I+2 G b_{6}, K^{4} C I+2 F b_{6}>, \\
&
\end{aligned}
$$




$$
\begin{aligned}
& J_{8}=<d_{1}, c_{1}, D F-C G, K^{2} I+G, \\
& G^{2} H-2 D G I-I^{2} b_{6} \text {, } \\
& D G H-2 D^{2} I+G^{2} I-H I b_{6} \text {, } \\
& G^{3}-G H b_{6}+D I b_{6}, F G^{2}-F H b_{6}+C I b_{6}, \\
& 2 D G^{2} I-G H^{2} b_{6}+D H I b_{6}+G I^{2} b_{6} \text {, } \\
& 2 C G^{2} I-F H^{2} b_{6}+C H I b_{6}+F I^{2} b_{6} \text {, } \\
& F G H^{2}-3 C G H I+2 C D I^{2}-F G I^{2} \text {, } \\
& F^{2} H^{2}-3 C F H I+2 C^{2} I^{2}-F^{2} I^{2}, \\
& K^{2} G H+2 D G+I b_{6}, K^{2} D H+2 D^{2}-G^{2}+H b_{6}, \\
& 2 D^{2} G^{2}-D^{2} H b_{6}+3 D G I b_{6}-H^{2} b_{6}^{2}+I^{2} b_{6}^{2} \text {, } \\
& K^{2} G^{2}-K^{2} H b_{6}-D b_{6} \text {, } \\
& K^{2} H^{2} b_{6}+2 D G^{2}+D H b_{6}+G I b_{6}, \\
& 4 D^{2} G I^{2}-G H^{3} b_{6}+D H^{2} I b_{6}+G H I^{2} b_{6}+2 D I^{3} b_{6} \text {, } \\
& 4 C D G I^{2}-F H^{3} b_{6}+C H^{2} I b_{6}+F H I^{2} b_{6}+2 C I^{3} b_{6} \text {, } \\
& 4 D^{3} G I-D^{2} H^{2} b_{6}+8 D^{2} I^{2} b_{6}-3 G^{2} I^{2} b_{6}-H^{3} b_{6}^{2}+4 H I^{2} b_{6}^{2} \text {, } \\
& K^{2} F H^{2}+3 C G H-2 C D I+F G I, K^{4} H+2 K^{2} D-b_{6}, \\
& 8 D^{3} I^{3}-G H^{4} b_{6}+D H^{3} I b_{6}-G H^{2} I^{2} b_{6}+8 D H I^{3} b_{6}+2 G I^{4} b_{6} \text {, } \\
& 8 C D^{2} I^{3}-F H^{4} b_{6}+C H^{3} I b_{6}-F H^{2} I^{2} b_{6}+8 C H I^{3} b_{6}+2 F I^{4} b_{6} \text {, } \\
& 8 D^{4} I^{2}-D^{2} H^{3} b_{6}+10 D^{2} H I^{2} b_{6}-H^{4} b_{6}^{2}+2 H^{2} I^{2} b_{6}^{2}-I^{4} b_{6}^{2}>\text {, } \\
& J_{9}=<d_{1}, c_{1}, F, C, K^{2} I+G, G^{2} H-2 D G I-I^{2} b_{6} \text {, } \\
& K^{2} G H+2 D G+I b_{6}, K^{4} H+2 K^{2} D-b_{6}>\text {, } \\
& J_{10}=<d_{1}, b_{6}, I, G, K^{2} H+D>\text {, } \\
& J_{11}=\left\langle d_{1}, b_{6}, I, G, K\right\rangle \text {, } \\
& J_{12}=\left\langle c_{1}, F, K\right\rangle \text {, } \\
& J_{13}=\langle I, G, F\rangle \text {. }
\end{aligned}
$$

\section{ACKNowledgements}

The first author is partially supported by a MINECO grant number MTM2014-53703-P and an AGAUR grant number 2014SGR 1204.

The second author is supported by Portuguese National Funds through FCT - Fundação para a Ciência e a Tecnologia within CAMGSD and the project PTDC/MAT/117106/2010. 


\section{REFERENCES}

[1] C. Christopher And C. Li, Limit cycles of differential equations. Advanced Courses in Mathematics. CRM Barcelona. Birkhäuser Verlag, Basel, 2007.

[2] C. J. Christopher AND N. G. Lloyd, On the paper of Jin and Wang concerning the conditions for a center in certain cubic system, Bull. London Math. Soc. 22 (1990), 5-12.

[3] I.A. García, Integrable zero-Hopf singularities and 3-dimensional centers. To appear in Proc. Roy. Soc. Edinburgh Sect. A.

[4] I.A. GARCíA AND C. VAlls, The three-dimensional center problem for the zero-Hopf singularity, Discrete Contin. Dyn. Syst. 36 (2016), 2027-2046.

[5] I. S. KuKLES, Necessary and sufficient conditions for the existence of acenter, Dokl. Akad. Nauk SSSR 42 (1944), 160-163 (in Russian).

[6] A.M. Liapunov, Problème général de la stabilité du mouvement, Ann. of Math. Studies 17, Princeton Univ. Press, 1949.

[7] J. Llibre, C. PAntazi And S. Walcher, First integrals of local analytic differential systems, Bull. Sci. Math. 136 (2012), 342-359.

[8] H. Poincaré, Mémoire sur les courbes définies par les équations différentielles, Oeuvres de Henri Poincaré, Vol. I, Gauthiers-Villars, Paris, 1051, pp. 95-114.

[9] V. G. Romanovski And D.S. ShafeR, The center and cyclicity problems: a computational algebra approach. Birkhäuser Boston, Inc., Boston, MA, 2009.

[10] C. Rousseau, D. Schlomiuk and P. Thibaudeau, The centers in the reduced Kukles system, Nonlinearity 8 (1995), 541-569.

[11] X. ZHANG, Analytic normalization of analytic integrable systems and the embedding flows, J. Differential Equations 244 (2008), 1080-1092.

[12] X. Zhang, Analytic integrable systems: Analytic normalization and embedding flows, J. Differential Equations 254, (2013), 3000-3022.

1 Departament de Matemàtica, Universitat de Lleida, Avda. Jaume II, 69, 25001 LLEIDA, SPAIN

E-mail address: garcia@matematica.udl.cat

2 Departamento de Matemática, Instituto Superior Técnico, Universidade Técnica de Lisboa, Av. Rovisco Pais 1049-001, Lisboa, PorTUGAL

E-mail address: cvalls@math.ist.utl.pt 